
SCIDiC
International Journal of Reproduction, Fertility \& Sexual Health (IJRFSH)

ISSN: $2377-1887$

\title{
Localization of Herbal Medicine Substances in Sperm that Increase Fertilization Rate During Mouse IVF
}

Short Communication

Tanaka $\mathrm{H}^{1 *}$, Haraguchi $\mathrm{T}^{1}$, Wada $\mathrm{M}^{1}$, Shoyama $\mathrm{Y}^{2}$

${ }^{1}$ Molecular Biology, Faculty of Pharmaceutical Sciences, Nagasaki International University, Huis Ten Bosch, Sasebo, Nagasaki, Japan.

${ }^{2}$ Pharmacognosy Laboratories, Faculty of Pharmaceutical Sciences, Nagasaki International University, Huis Ten Bosch, Sasebo, Nagasaki, Japan.

\section{Abstract}

Isoliquiritigenin and formononetin are flavonoids in licorice that significantly improve in vitro fertilization rates in mice. However, how these substances act on sperm is unknown. Since both are fluorescent substrates, the sperm in medium containing these substances was observed using a fluorescence microscope. We observed strong signals in the postacrosomal and midpiece regions. Various physiological activities of these molecules have been reported. It is thought that isoliquiritigenin and formononetin contribute to sperm motility after ejaculation and acquisition of fertility. The localization of these molecules in sperm would help to elucidate their function. The results will contribute to human infertility treatments.

Keywords: Testis; Spermatogenesis; Licorice; Infertility; Flavonoid.

\section{Introduction}

One in five couples in Japan is infertile, and the male partner is the cause in $50 \%$ of cases [1]. Varicocele results in male infertility, but many of the causes are unknown. Apart from abnormalities in the $\mathrm{Y}$ chromosome, gene polymorphisms and single nucleotide polymorphisms are considered the cause of male infertility cases [2], but the only treatment option available is assisted reproductive technology (ART). Although ART is a very effective treatment for infertility, it is a physically, economically, and mentally burdensome process. Development of easier treatments and/ or therapeutic drugs that can complement ART is expected. We screened natural products to improve fertilization rates by adding each product to sperm preincubation medium prior to in vitro fertilization (IVF). We found that a water-soluble component of licorice that has been used in at least $70 \%$ of traditional Chinese medicine formulas for various diseases, including gastrospasm, stomachache, sore throat, and gastric and duodenal ulcers [3], increased the fertilization rate. After activity-guided separation we determined two active flavonoids, isoliquiritigenin and formononetin [4]. It will be important to determine how their molecules, which may pass through the sperm cell membrane, act on sperm. Therefore, we observed these fluorescent molecules with a fluorescence microscope in culture medium containing sperm.

\section{Materials and Methods}

\begin{abstract}
Animals
Ten-week-old ICR mice were purchased from Japan SLC (Shizuoka, Japan). The animals were killed by cervical dislocation just before the experiments. All animal experiments conformed to the Guide for the Care and Use of Laboratory Animals and were approved by the Institutional Committee of Laboratory Animal Experimentation (Nagasaki International University, Nagasaki, Japan).
\end{abstract}

\section{Sperm and Egg Collection}

Mature caudal epididymal sperm $\left(\sim 8 \times 10^{\circ}\right)$ from each mouse were incubated in $200 \mu$ l of human tubal fluid (HTF) medium without bovine serum albumin (BSA) $\left(\right.$ LifeGlobal ${ }^{\circledR}$ medium; IVFonline, Guilford, CT, USA) covered with paraffin oil. After 5 min, each sperm suspension was transferred to HTF medium with $0.4 \%$ BSA and $0.02 \mathrm{mg} / \mathrm{ml}$ isoliquiritigenin or formononetin. The sperm were observed under a fluorescence microscope after 30 $60 \mathrm{~min}$.

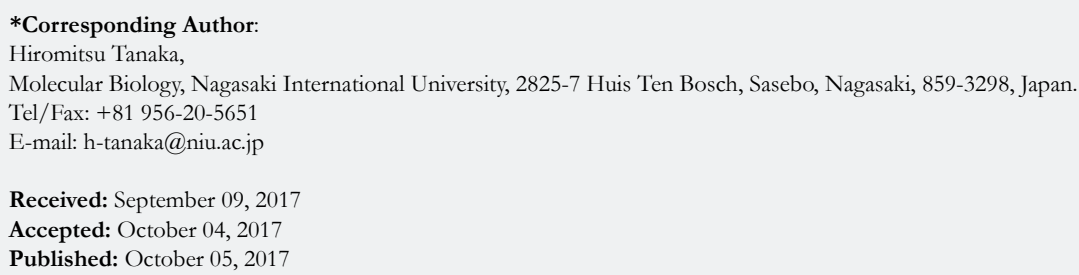

Citation: Tanaka H, Haraguchi T, Wada M, Shoyama Y. Localization of Herbal Medicine Substances in Sperm that Increase Fertilization Rate During Mouse IVF. Int J Reprod Fertil Sex Health. 2017;4(5):113-115. doi: http://dx.doi.org/10.19070/2377-1887-1700020

Copyright: Tanaka $\mathbf{H}^{\circ}$ 2017. This is an open-access article distributed under the terms of the Creative Commons Attribution License, which permits unrestricted use, distribution and reproduction in any medium, provided the original author and source are credited. 
Cumulus-oocyte complexes were obtained from the ampullae of uterine tubes in hormonally controlled female mice and transferred to dishes, each containing a 200-ml drop of HTF medium covered with paraffin oil, under a stereomicroscope. Eggs were transferred to the HTF medium with $0.4 \%$ BSA and $0.02 \mathrm{mg} /$ $\mathrm{ml}$ of each substance and were observed under a fluorescence microscope $30 \mathrm{~min}$ after insemination.

\section{Results and Discussion}

Sperm of BALB/cA mice have a disrupted morphology and their IVF efficiency is low [5]. However, pre-incubating the sperm of $\mathrm{BALB} / \mathrm{cA}$ mice with isoliquiritigenin or formononetin in medium significantly improves the fertilization rate [4]. To analyze the mechanism of action, the sperm in medium containing these molecules was observed using a fluorescence microscope, as these substances are fluorescent. Strong signals were obtained in the post-acrosomal and midpiece regions (Figure 1). To investigate the permeability of the cell membrane to these molecules, we also analyzed staining of eggs in medium with isoliquiritigenin or formononetin, because it is difficult to observe swimming sperm using laser microscopy. The sphere fluoresced evenly across the cell (Figure 2), indicating that isoliquiritigenin and formononetin were incorporated into the cells. It is considered that the postacrosomal region and mid-piece of the sperm were strongly stained because these regions are cytoplasm rich. These substances have been reported to exhibit antioxidative and antitumor activities [6]. Isoliquiritigenin affects cell viability, and may increase the fertility rate under IVF conditions. It also affects mitochondrial membranes [7], so it may be confirmed as an effective energy producer under IVF conditions. Isoliquiritigenin is a positive allosteric modulator of the gamma aminobutyric acid type A receptor, which induces hypnotic effects when administered orally to mice [8]. It also has an antagonistic action on the serotonin 5-HT2C receptor in rats [9]. These results indicate that isoliquiritigenin is easily incorporated into the body. In the future, we will determine whether isoliquiritigenin reacts with specific molecules in sperm using an overlay assay.

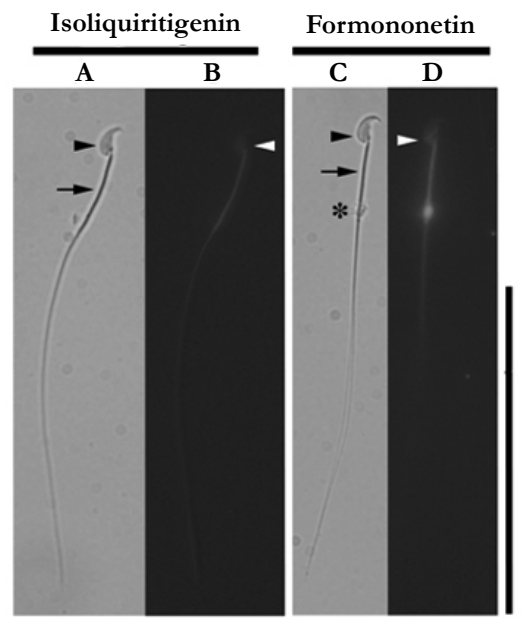

Figure 1. Fluorescence imaging of mouse sperm in pre-incubation medium with isoliquiritigenin or formononetin. Signals were observed intensely in the midpiece (arrows), postacrosomal region (arrowheads), and residual body (star). Bar = $50 \mu \mathrm{m}$.
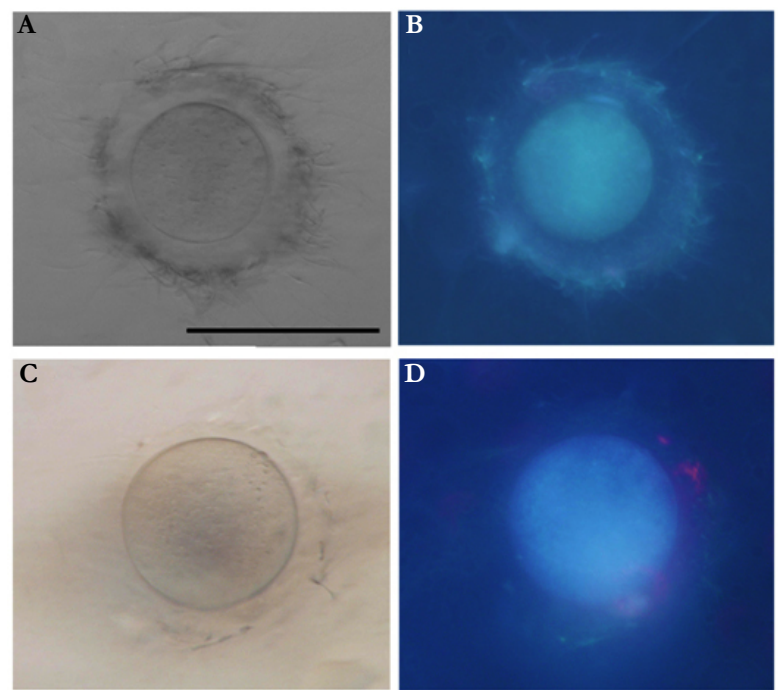

Figure 2. Fluorescence imaging of mouse egg with isoliquitigenin or formation. Bright field (A and C) and fluorescence of DAPI (4',6-diamidino-2-phenylindole) Emission filter (B and D) imaging of a mouse egg in the medium contained propidium iodide with isoliquiritigenin (A and $B$ ) and formononetin (C and D) on 30 to 60 minutes after insemination. Blue signals were observed as a content of spheroidal form. Red signals showed dying sperm on zona pellucida. $\mathrm{Bar}=100 \mu \mathrm{m}$. 


\section{References}

[1]. Japan Society for Reproductive Medicine, Funinshou Q \& A; 2013. (www. jsrm.or.jp/document/funinshou_qu.pdf)

[2]. Tanaka H, Hirose M, Tokuhiro K, Tainaka H, Miyagawa S, Tsujimura A, et al. Molecular biological features of male germ cell differentiation. Rep Med Biol. 2007 Mar 6(1):1-9.

[3]. Tung HN, Shoyama Y, Wada M, Tanaka T. Improved In Vitro Fertilization Ability of Mouse Sperm Caused by the Addition of Licorice Extract to the Preincubation Medium. The Open Reprod Sci J. 2015;6:1-7.

[4]. Tung HN, Shoyama Y, Wada M, Tanaka H. Two activators of in vitro fertilization in mice from licorice. Biochem Biophys Res Commun. 2015 Nov 13; 467(2):447-450. PubMed PMID:26392313.

[5]. Kishikawa $\mathrm{H}$, Tateno $\mathrm{H}$, Yanagimachi R. Chromosome analysis of BALB/c mouse spermatozoa with normal and abnormal head morphology. Biol Reprod. 1999 Sep; 61(3):809-812. PubMed PMID:10456861.
[6]. Peng F, Du Q, Peng C, Wang N, Tang H, Xie X, et al. A Review: The Pharmacology of Isoliquiritigenin. Phytother Res. 2015 Jul; 29(7): 969-977. PubMed PMID:25907902

[7]. Jung JI, Lim SS, Choi HJ, Cho HJ, Shin HK, Kim EJ, et al. Isoliquiritigenin induces apoptosis by depolarizing mitochondrial membranes in prostate cancer cells. J Nutr Biochem. 2006 Oct;17(10): 689-696. PubMed PMID: 16517140

[8]. Cho S, Kim S, Jin Z, Yang H, Han D, Baek NI, et al. Isoliquiritigenin, a chalcone compound, is a positive allosteric modulator of GABAA receptors and shows hypnotic effects. Biochem Biophys Res Commun. 2011 Oct 7; 413(4): 637-642. PubMed PMID:21945440.

[9]. Arai T, Maejima Y, Muroya S, Yada T. Rikkunshito and isoliquiritigenin counteract 5-HT-induced 2C receptor-mediated activation of pro-opiomelanocortin neurons in the hypothalamic arcuate nucleus. Neuropeptides. 2013 Aug; 47(4): 225-230. PubMed PMID:23756052. 